\title{
Análisis y selección de datos para la solución de problemas profesionales en la carrera de Ingeniería Automotriz
}

\section{Analysis and selection of data for the solution of professional problems in the career of Automotive Engineering}

Virgilio Lucas Ramos Rivero

Adolfo Juan Peña Pinargote

Universidad Internacional del Ecuador, Ecuador

Autor para correspondencia: viramosri@uide.edu.ec; ajpena@uide.edu.ec

Fecha de recepción: 1 de agosto de 2018 - Fecha de aceptación: 01 de diciembre de 2018

Resumen: En el desarrollo de las actividades académicas, y en particular en aquellas destinadas a la solución de problemas en los distintos niveles de enseñanza, es común presentar el enunciado de los problemas y los datos que sirven de base para su solución. Este enfoque es utilizado tradicionalmente no sólo por los docentes sino también en la mayoría de los libros de texto que se emplean en las diferentes asignaturas. Si bien es cierto que la lectura e interpretación del enunciado de un problema y la habilidad para vincular los datos con los conceptos y con las relaciones matemáticas para el proceso de su correcta solución, constituyen un elemento esencial de vinculación de la teoría con la práctica, no deja de ser a juicio de los autores del presente trabajo, un enfoque académico que no en todos los casos responde a la realidad, particularmente en el ejercicio de una carrera universitaria. Es típico en el ejercicio de una profesión, en particular aquellas de carácter tecnológico, que el profesional deba esclarecer entre un gran número de elementos o datos, aquellos verdaderamente útiles para la solución de un problema, de modo que no siempre podrá contar con datos a priori y tendrá que realizar un análisis preciso para separar lo superfluo o irrelevante de lo verdaderamente importante. Este trabajo presenta una alternativa didáctica para la solución de problemas vinculados con la profesión por parte de los estudiantes, en el contexto de las asignaturas de formación profesional en la Carrera de Ingeniería Automotriz. Palabras claves: tecnológico; problemas profesionales; aprendizaje significativo; constructivismo; selección de datos

\begin{abstract}
In the development of academic activities, particularly those destined to problemsolving tasks throughout different educational levels, it is a common occurrence to present problem statements in addition to the data required as a basis to find a solution. This approach is used traditionally, not only by teachers but also textbooks used in a variety of subject areas, due to the advantages it offers. While the reading, interpretation of the problem statement, and the ability to associate data to the concepts and mathematical relations for its proper solution, constitute an essential element in the connection of theory to practice, it does persist to be, in the researchers' judgement, an academic approach that not in every case responds to real-life, especially in the practice of the profession. It is typical in the exercise of the profession, especially in those of technological character that the professional must establish among a great number of elements or data, those which are actually useful for the solution of a problem. This in contrast to reality where
\end{abstract}


there is rarely a possibility to get data beforehand and it is required to perform a precise analysis to separate superfluous or irrelevant data from what is truly relevant. The objective of this work is to show a didactic alternative for the solving of profession-related problems by students in the context of the professional training subjects in the Automotive Engineering degree.

Key words: technology; professional problem-solving; significant learning; constructivism; data selection

\section{Introducción}

La enseñanza de la ingeniería desde su surgimiento ha estado condicionada por diferentes cambios que la han hecho evolucionar y a la vez enriquecerse. Uno de los aspectos que la han hecho evolucionar a lo largo del tiempo, ha sido la preocupación por la formación de un ingeniero acorde con las necesidades del entorno en que vive y se desenvuelve, así como la manera en que debe enfrentar dicha formación.

En correspondencia con lo anterior, una de las principales estrategias utilizadas en la formación de un ingeniero automotriz es la de enfrentar al estudiante de la carrera en mención, al análisis de diferentes situaciones vinculadas con su labor y particularmente a la solución de problemas profesionales. Esta forma de relación de los contenidos académicos con la práctica es sin lugar a dudas una herramienta de gran valor didáctico para el logro de aprendizajes significativos.

No obstante, lo anterior, la forma de presentar dichos problemas, en particular en las asignaturas de praxis profesional de la carrera de ingeniería automotriz, puede o no incidir favorablemente en la preparación del estudiante para enfrentar y resolver de manera efectiva los problemas que se le presenten durante su labor ingenieril.

Como parte de la forma de presentación del problema se encuentran la información involucrada en el mismo, la claridad de su redacción, y su sistematización en correspondencia con las distintas etapas de formación del ingeniero.

En el presente trabajo se hace una valoración de los enfoques utilizados en el planteamiento de los problemas profesionales y se proponen algunas alternativas para el planteamiento de dichos problemas, teniendo en cuenta el análisis y selección de datos por parte de los estudiantes.

Para el presente trabajo los autores han definido el problema en los siguientes términos: ¿Cómo incide el análisis y selección de datos en la solución de problemas profesionales por parte de los estudiantes, en el logro de aprendizajes significativos en las asignaturas de formación profesional de la carrera de ingeniería automotriz?

Relacionado con el problema anterior se planteó como objetivo general: Valorar la incidencia del análisis y selección de datos en la solución de problemas profesionales por parte de los estudiantes, en el logro de aprendizajes significativos en las asignaturas de formación profesional de la carrera de ingeniería automotriz.

\section{Métodos}


El presente trabajo se enmarca en una investigación que se desarrolla actualmente en la Facultad de Ingeniería Automotriz de la UIDE, titulada "Incidencia de los métodos para la solución de problemas de la profesión en las asignaturas de formación profesional, en el logro de aprendizajes significativos de los estudiantes de la carrera de Ingeniería Automotriz de la UIDE, Extensión Guayaquil, de abril de 2018 a marzo de 2019. Propuesta de una metodología basada en el constructivismo para la solución de problemas profesionales.

Los métodos de carácter teórico utilizados fueron:

- La observación

- El método analítico - sintético

- El método inductivo deductivo

Las técnicas de recolección de datos empleadas fueron:

- Revisión y análisis documental

- El análisis de las experiencias de los autores en el ejercicio de la docencia

\section{Participantes:}

Estudiantes de ingeniería de distintos niveles de la carrera que cursan asignaturas de la praxis profesional. Por su importancia en relación con el presente trabajo, se definieron los siguientes términos o variables:

\section{Aprendizaje significativo:}

El aprendizaje significativo s aquel que se caracteriza por relacionar el nuevo material de estudio con las ideas ya existentes en la estructura cognitiva del estudiante. Por consiguiente, la eficacia de tal aprendizaje está en función de su carácter significativo, y no en las técnicas memorísticas (Pimienta Prieto, 2007).

Para ello, los requisitos básicos son:

a) Disposición del estudiante para aprender y dar sentido a lo que aprende, así como disponer de los conocimientos previos que le permitan aprender lo nuevo de forma significativa.

b) Que el material de estudio sea potencialmente de modo que permita al estudiante establecer una relación sustantiva con los conocimientos e ideas que ya posee.

c) Una actitud activa del docente mediador tendiente a lograr un aprendizaje significativo en sus estudiantes

Grado de significación del aprendizaje. 
Medida en que los estudiantes han sido capaces de construir con sus propios recursos cognitivos y la mediación del docente, interpretaciones significativas y valiosas de los contenidos estudiados y atribuir un valor funcional a dichas interpretaciones en función de las competencias previstas en el micro currículo de la asignatura cursada (Ramos Rivero, 2013).

\section{Aprendizaje basado en problemas:}

El aprendizaje basado en problemas se fundamenta en el principio de usar problemas como punto de partida para la adquisición e integración de nuevos conocimientos (Barrows, 1986)

\section{Problemas profesionales:}

Son aquellos que se presentan en la actividad del profesional, dados por la necesidad que tiene la sociedad y que requieren de la actuación del profesional para satisfacerlas. También pueden definirse como situaciones objetivas presentes en la sociedad, pero que son analizadas, caracterizadas, valoradas como problemas por aquel sujeto que siente la necesidad de resolverlos (Alvarez de Zayas, 2001).

\section{Fundamentación}

El constante desarrollo de la humanidad desde el punto de vista socio cultural y particularmente en lo referente a ciencia y la tecnología, demanda un constante perfeccionamiento y adecuación de los sistemas educativos a esas nuevas condiciones de vida y de desarrollo.

El sistema de educación superior debe sufrir cambios trascendentales aumentando su capacidad de adaptación a las nuevas condiciones de desarrollo, diversificándose institucionalmente en sus estructuras, en sus estudios y formas de organización, poniendo además al día los conocimientos y competencias, para así mejorar la cultura general de la sociedad, así como de los individuos en particular (UNESCO, 1998).

Por otro lado, la creciente importancia concedida a la educación científica y las dificultades encontradas para su extensión a la generalidad de los futuros ciudadanos y ciudadanas ha impulsado la investigación en torno a la educación científica y tecnológica (Simpson, 1994).

El mundo en constante cambio demanda profesionales preparados para enfrentarse a nuevos retos en el campo laboral. Profesionales con iniciativa, capacidades comunicativas, argumentativas, asertivos y con espíritu investigativo es la tendencia actual. Estas aptitudes se forman y consolidan en las aulas universitarias y a través de metodologías didácticas centradas en el estudiante (Mendoza, 2006).

En relación con lo anterior, si bien es cierto que en el Sistema de Educación Superior del Ecuador se han evidenciado transformaciones profundas tendientes a mejor la calidad de los futuros profesionales, desde el punto de vista del tratamiento metodológico de los contenidos de 
carácter técnico y las metodologías de la enseñanza en el contexto de las carreras tecnológicas, queda aún un gran campo por investigar.

Incidencia de la solución de problemas en el logro de aprendizajes significativos

Existen diversas metodologías que buscan acercar al estudiante a la solución de problemas del mundo real. Una de ellas es el Aprendizaje Basado en Problemas, ABP, la cual permite centrar el aprendizaje en el estudiante, e introducir en la enseñanza problemas de diferentes tipos y grados de complejidad, incluyendo los de carácter abierto que, desde el punto de vista de una carrera universitaria ingenieril, son más próximos al futuro desempeño profesional del estudiante.

Según Restrepo un problema abierto, no estructurado o brunerianos, son problemas intencionalmente mal estructurados que no orientan de forma clara la solución

El aprendizaje basado en problemas es un método de enseñanza aprendizaje centrado en el estudiante que adquiere conocimientos, habilidades y actitudes a través de situaciones de la vida real (Bernabeu, 2018)

Dicho aprendizaje es un proceso complejo que debe responde a una concepción de un aprendizaje más integrador en función de alcanzar las competencias requeridas por el futuro ingeniero.

Se han dado múltiples definiciones para un problema, Mazario nos da una definición aplicada a las matemáticas, pero que bien se ajusta a otros contextos, donde "Un problema es una situación o dificultad prevista o espontánea, con algunos elementos desconocidos para el sujeto, pero capaz de provocar la realización de acciones sucesivas para darle solución" (Mazario, 2009).

La resolución de problemas no sólo da al estudiante la posibilidad de aplicar sus conocimientos en la solución de situaciones prácticas, contribuyendo a que el aprendizaje sea significativo, sino que, para el profesor, ellos son uno de los métodos más efectivos de comprobar cuán profundamente el estudiante comprende la asignatura y de verificar si su conocimiento es sólo una acumulación de lo estudiado de memoria (Kapitsa, 1985).

El aprendizaje basado en problemas posee las siguientes características (Universidad Politécnica de Madrid, 2008):

- Responde a una metodología centrada en el alumno y en su aprendizaje.

- Los alumnos trabajan en pequeños grupos que deben ser en un número entre cinco y ocho estudiantes.

- Dicha metodología favorece la posibilidad de interrelacionar distintas materias o disciplinas académicas.

- Este aprendizaje puede utilizarse como estrategia dentro del proceso de enseñanza aprendizaje, aunque también es posible aplicarlo en una asignatura durante todo el curso académico. 
Además, al enseñar a los estudiantes se puede con la ayuda de los problemas, educar y poner de manifiesto el pensamiento científico creador (Kapitsa, 1985).

Entre las posibilidades que ofrece el aprendizaje basado en problemas y su contribución al logro de aprendizajes significativos se pueden mencionar las siguientes:

- Enfrenta al sujeto con la realidad, la reconoce y adopta una postura frente a ello.

- Provoca una mayor actividad en el estudiante al intentar resolver la situación al buscar respuestas científicas y tecnológicas.

- Favorece la formación de un pensamiento creativo, al tratar de hallar la solución desde lo diferente, al encontrar y establecer relaciones, consolidaciones y respuestas lógicas.

- Al trabajar en grupo, estimula el sentido de colaboración.

- Propicia la permanente duda, la actitud hacia la investigación científica como modo de bordar la realidad y llegar a resultados objetivos en su perfeccionamiento.

- Integra y sistematiza conocimientos y habilidades.

- Desarrolla un mayor interés por la profesión, al promover un aprendizaje significativo donde se manifiesten sentimientos de pertenencia y satisfacción por la labor realizada.

En relación con el docente en el aprendizaje basado en problemas, el estudiante deja de tener un rol pasivo frente a este y toma un papel protagonista en la construcción de su aprendizaje, mientras que el docente se convierte en un guía, tutor y faci8litador del aprendizaje cuando lo necesitan (Universidad República Dominicana, 2015).

El A. B. P. se debe de caracterizar por ser flexible, desarrollado desde la praxis, participativo, interdisciplinario, investigativo e integrado (Correa Betancourt, 2006).

Los psicólogos cognitivos están de acuerdo en que existen destrezas generales para la solución de problemas, pero también lo están en el hecho de que el proceso es muy dependiente del conocimiento mínimo requerido para encontrar la solución.

De acuerdo con el tipo de problema que se confronta, dicho proceso de solución puede clasificarse en tres esquemas:

1. Un esquema basado en el grado de definición del problema, relacionado estrechamente con la estrategia requerida;

2. Estrategias relativamente bien estructuradas para problemas bien definidos; y

3. Problemas deficientemente estructurados o pobremente definidos que requieren un enfoque concentrado en su determinación y en las metas. Para problemas con grados intermedios de definición se pueden aplicar estrategias de pasos múltiples.

Es importante señalar que la estrategia de solución está condicionada por el tipo de problema. Existen diferentes tipologías de problemas.

Una tipología a considerar es la de clasificar los problemas en tres grupos denominados: 
1. De carácter propedéutico los cuales están dirigidos a sistematizar conocimientos y habilidades, así como a solucionar insuficiencias de orden teórico y práctico.

2. De tipo situacional que están orientados a resolver situaciones específicas del proceso profesional como puede ser la necesidad de una reparación en el circuito eléctrico, la explotación de un equipo caminero, la solución de un conflicto laboral, diagnosticar el estado de un motor de combustión interna, diagnosticar fallos en el sistema de arranque de un vehículo a gasolina entre otros.

3. De tipo proyectivo, los cuales están encaminados a ofrecer una solución a insuficiencias de mayor complejidad que exigen un alto nivel de integración de conocimientos y habilidades; deben conducir a dar un aporte de orden teórico o práctico, como pueden ser el análisis comparativo de calidades de aceites, la modificación de un sistema de suspensión, la adecuación de un vehículo para trabajar con diferentes tipos de combustibles, entre otras.

En el de tipo situacional, el nivel de información inicial (datos) puede ser completo, nulo o parcial, lo cual es aplicable según el nivel de la carrera de ingeniería en que se encuentre el estudiante.

En el tipo de problemas proyectivo, el nivel de información inicial facilitado al estudiante para resolver el problema puede ser nulo o incompleto, de modo que el estudiante deberá lograr un mayor nivel de comprensión de los términos del problema y gestionar los datos, e incluso inventarlos de ser necesario. Este caso se corresponde más con la realidad del ejercicio de la profesión ingenieril.

A diferencia de los problemas que se plantean en muchos casos o habitualmente a los estudiantes de ingeniería durante su formación, los problemas reales no están estructurados, ni tienen una finalización clara. Algunas veces, no están disponibles todos los datos para su resolución, o estos están confundidos con otros detalles sin importancia. En estos casos, se hace necesario ordenar todo un conglomerado de información, para identificar, que partes de ésta se necesitan para resolver el problema concreto. Por otro lado, el estudiante debe localizar la información básica necesaria para lograr la solución.

Precisamente teniendo en cuenta el principio didáctico del aprendizaje de ir de lo fácil a lo complejo y de ir logrando progresivamente la independencia cognoscitiva del estudiante de ingeniería, en las primeras etapas de la carrera el nivel de información recibido por el estudiante por parte del docente, su carácter tutorial y facilitador, deberá ser mayor, disminuyendo progresivamente en la misma medida que el estudiante avanza en sus estudios.

Esta forma de enfocar y dosificar el nivel de información de los problemas contribuye al desarrollo de la capacidad de razonamiento del estudiante, así como a fortalecer y ampliar su estructura cognitiva. Al interrelacionar los contenidos anteriores con los nuevos involucrados en el problema y darle un significado funcional, contribuye a que el aprendizaje sea más significativo. 
Es imprescindible que el estudiante al graduarse haya alcanzado un sustancial nivel de independencia.

Eso no niega el hecho de que una vez graduado el ingeniero novato no tenga que pasar por un período de adaptación que puede durar un mayor o menor tiempo, dependiendo de las condiciones laborales y del propio recién graduado.

\section{Formación en la carrera de ingeniería automotriz}

La labor de formación en la Carrera de Ingeniería en Mecánica Automotriz de la Universidad Internacional del Ecuador, Extensión Guayaquil, está encaminada el logro de aprendizajes significativos en función del Perfil Profesional, aspecto que actualmente se perfecciona, no sólo a nivel de documentos curriculares, sino en los distintos procesos de aprendizaje que se desarrollan en las diferentes asignaturas de formación profesional de la carrera.

Cabe señalar que, durante el proceso de formación en la mencionada carrera, se han materializado en la práctica y en cada uno de los años de estudio, un considerable número de proyectos que han permitido solucionar variados e importantes problemas relacionados con el área automotriz. Entre los distintos problemas abordados se pueden mencionar la construcción de equipos de laboratorio, módulos de simulación en el área de autotrónica, hidráulica, motores a diésel, a gasolina, flujómetro, iluminación del vehículo, inyección electrónica, transmisiones mecánicas y automática, sistemas de freno hidráulico y neumático, entre otros, así como la adaptación de sistemas de combustibles y diseño y de algunos componentes vehiculares.

Es importante señalar que el desarrollo de los referidos proyectos ha tenido un carácter interdisciplinario, lo que ha permitido integrar los contenidos de diferentes asignaturas de la carrera.

Lo anterior es una muestra de cómo el aprendizaje ha estado encaminado en todo momento a vincular la teoría con la práctica mediante la solución de problemas de diferente complejidad y relacionados con la profesión del ingeniero automotriz, presentados generalmente en forma de proyectos.

No obstante, lo anterior, resulta indispensable en la referida carrera, tener en cuenta como parte de la estructura de los problemas que se plantean a los estudiantes, el aumento de su nivel de complejidad teniendo en cuenta el mayor o menor suministro de información para llegar a la solución y lograr de esta forma un acercamiento progresivo a las condiciones reales de trabajo. Por otro lado, si bien es cierto que los proyectos contribuyen con la formación del estudiante de ingeniería, no siempre su actividad estará dirigida en este sentido.

Es bien conocido el gran valor que tiene la resolución de problemas al estudiar las ciencias básicas, tales como la matemática, la física y la química lo que se refleja en la amplia bibliografía que trata sobre este tema. En contraposición, en las ciencias técnicas, no puede decirse lo mismo y en lo que se refiere a las ciencias de la ingeniería en particular, trabajar con 
algoritmos en la solución de problemas, es lo más común. Este es también el caso de las asignaturas de formación profesional de la carrera de ingeniería automotriz

Desde el punto de vista de la dificultad que ofrece el problema y el tipo de actividad mental encaminada a la superación, todos los problemas... pueden subdividirse en dos tipos: Los algoritmos y los heurísticos. Respecto a los problemas algorítmicos señala requieren la aplicación de un algoritmo ya preparado y asimilado por los estudiantes incluyendo operaciones precisas y un orden lógico, mientras que los heurísticos no indican algoritmos de solución de modo que hay que hallar el procedimiento de solución del problema dado (Majmutov, 1983)

En el trabajo con algoritmos, el estudiante se familiariza con problemas tipo, para cuya solución existe una secuencia de pasos previamente establecida. Si bien es cierto que en una etapa inicial de formación este tipo de soluciones contribuye a organizar y sistematizar el pensamiento lógico de los estudiantes y resolver ciertos problemas de la profesión con esas características, en etapas superiores de formación del estudiante de ingeniería se debe lograr un acercamiento cada vez mayor al planteamiento de problemas más complejos donde la información y el método de solución, no resulten tan evidentes.

Es importante señalar que en la rama de la ingeniería automotriz y a modo de ejemplo, son innumerables los problemas que se manifiestan en forma de fallos en los diferentes sistemas de los vehículos automotores y que son diagnosticados de forma empírica, basado en la experiencia profesional o utilizando herramientas y o equipos de diagnóstico, por lo que resulta indispensable preparar a los futuros ingenieros de esta rama en la solución de este tipo de problemas.

Siguiendo con el ejemplo de los problemas de tipo diagnóstico, el suministro de datos a los estudiantes debe dosificarse gradualmente, de modo que en forma creciente dichos problemas vayan teniendo un carácter más abierto y por lo tanto cada vez más cercano a su actividad profesional.

Aspectos importantes para enunciar e instrumentar problemas profesionales en el proceso de aprendizaje

- Todas las asignaturas de la malla curricular de la carrera deben tributar al desarrollo de las competencias del perfil profesional de la misma, por lo que deben estructurar problemas relacionados con la profesión que contribuyan a su vez al logro de dichas competencias y resultados de aprendizaje correspondientes.

- Los problemas deben estar expresados en un lenguaje fácilmente comprensible para los alumnos incluyendo las explicaciones adicionales verbales y gráficas adecuadas, teniendo en cuenta el lenguaje científico más próximo al nivel cognoscitivo del alumno (Selles Cantó, 2011)

- Los datos o pistas precisas deberían graduarse para el hallazgo de la solución de modo creciente. Esto es al inicio del curso los problemas contendrían los datos precisos para su solución, pero de un modo gradual podrían irse eliminando de modo que el alumno hubiera de buscarlos por su cuenta, preguntarlos al profesor o inventarlos. Esto 
propiciaría que el estudiante tome conciencia de que en la práctica de la profesión los datos iniciales se desconocen o sólo se conocen parcialmente (Selles Cantó, 2011).

- Los problemas deberán estar vinculados a situaciones reales del ejercicio de la profesión con e información fidedigna.

- Se debe dar acceso en la solución de problemas a la consulta de manuales y bibliografía en general incluyendo el uso de Internet, de modo que el estudiante complete la información necesaria para la solución del problema. Progresivamente el estudiante deberá ganar en independencia a la hora de localizar las fuentes bibliográficas y la información correspondiente como parte de estas fuentes.

- Se debe pasar progresivamente de la búsqueda de las fórmulas que permitan encontrar el resultado correcto, a otras formas más coherentes con los procedimientos de la ciencia y en particular con el método científico.

Ejemplos de estructuración de problemas considerando la dosificación de datos proporcionados a los estudiantes para su solución.

Veamos a continuación un par de ejemplos a considerar en ls asignatura Equipos de diagnóstico automotriz:

1) Problema con datos que orientan al estudiante

Un conductor en un día normal por la mañana quiere encender su vehículo, pero se da cuenta que el mismo no enciende y a su vez detecta en el tablero que hay una señal de advertencia que representa una batería. Se sabe que dicha señal corresponde al sistema de carga y arranque del vehículo. Diagnosticar y solucionar el problema.

2) Problema sin datos por lo que el estudiante no cuenta con información alguna para la solución del problema.

El auto no enciende.

En ambos tipos de problemas la secuencia de solución es la misma, sin embargo, el nivel de información suministrada es diferente, de acuerdo con el nivel de conocimiento y habilidades alcanzados por el estudiante.

La solución común del problema sería la siguiente:

1. Revisar la batería y el motor de arranque

2. Pide que prueben la batería.

3. Pídele a alguien que conecte cables de refuerzo a la batería si el motor parece estar volviéndose más lento de lo normal.

4. Observa la condición de la conexión del cable de la batería.

5. Decide si el motor de arranque es el problema.

\section{Parte 2}

Revisar el sistema de combustible 
1. Asegúrate de tener combustible en el tanque.

2. Muchos motores de inyección de combustible tienen un puerto de prueba en la línea de entrega de combustible.

3. Revisa el filtro de combustible para asegurarte de que esté dejando pasar el combustible.

\section{Parte 3}

1. Probar el sistema de encendido Busca componentes dañados o sueltos en el sistema de encendido

2. Busca componentes dañados o sueltos en el sistema de encendido.

\section{Conclusiones}

Una de las principales estrategias utilizadas en la formación de un ingeniero automotriz es la solución de problemas, que puede ser una herramienta de gran valor didáctico para el logro de aprendizajes significativos.

Como parte de la forma de presentación del problema se encuentran la información involucrada en el mismo, la claridad de su redacción, y su sistematización en correspondencia con las distintas etapas de formación del ingeniero.

La resolución de problemas da al estudiante la posibilidad de aplicar sus conocimientos en la práctica contribuyendo a que el aprendizaje sea significativo mientras que, para el profesor, constituye uno de los métodos más efectivos para comprobar cuán profundamente el estudiante comprende la asignatura y de verificar la solides de sus conocimientos.

La forma de enfocar y dosificar el nivel de información de los problemas contribuye al desarrollo de la capacidad de razonamiento del estudiante, así como a fortalecer y ampliar su estructura cognitiva de modo que al interrelacionar los contenidos anteriores con los nuevos involucrados en el problema y darle un significado cada vez más funcional, contribuye a que el aprendizaje sea más significativo.

\section{Bibliografía}

Alvarez de Zayas, C. (2001). El Diseño Curricular. La Habana: Pueblo y Educación.

Barrows, H. (1986). Taxonomy of problem-based learning methods. Medical Education, 481-486.

Bernabeu, M. D. (2018). Aprendizaje basado en problemas. Educrea.

Correa Betancourt, C. (2006). Aprendizaje basado en problemas, una experiencia novedosa en la enseñanza de la ingeniería. Educación en Ingeniería, 45-51.

Kapitsa, P. (1985). Experimento, teoría y práctica. Moscu: Mir. M.a. (s.f.). 
Majmutov, M. (1983). La enseñanza problémica. La Habana: Pueblo y Educación.

Mazario, T. (2009). La resolución de problemas: Un reto para la educación contemporánea. La Habana: Universitaria.

Mendoza, M. (2006). Aprendizaje basado en problemas. Innovación Educativa, 1-12. Otros, M. S. (s.f.). web.ua.es. Recuperado el 21 de octubre de 2018, de https://web.ua.es/es/ice/jornadas-redes-2011/documentos/posters/183924.pdf

Pimienta Prieto, J. (2007). Metodología Constructivista, guía para la planeación docente. México: Pearson Educación.

Ramos Rivero, V. L. (2013). Incidencia de la evaluación de las asignaturas de formación profesional en la valoración del grado de significación de los aprendizajes de los estudiantes de la carrera de ingeniería en mecánica automotriz de la UIDE, Extensión Guayaquil, año 2013tomotriz. Guayaquil.

Selles Cantó, M. (2011). Los problemas en ingeniería. Obtenido de https://web.ua.es/es/ice/jornadas-redes-2011/documentos/posters/183924.pdf.

Simpson, R. (1994). Handbook of Reasesrch on Science Teaching and Learning.

UNESCO. (1998). Conferencia Mundial. New York.

Universidad Politécnica de Madrid. (2008). Aprendizaje basado en problemas. Servicios de Innovación Educativa.

Universidad República Dominicana. (2015) ¿Qué es el aprendizaje basado en problemas? Universia. 